$s$ must be commutative with at least one of the substitutions of $H$ besides identity. If a transitive group of degree $n$ is transformed into itself by any substitution in the same letters and if the degree of this substitution is not $n-1$, then it must be commutative with at least one of the substitutions of the transitive group besides identity.

7. The object of Professor Allardice's note on the cyclide of Dupin was to show that, by means of a transformation originally due to Laguerre (see Darboux, Théorie des surfaces, volume 1, page 253), a circle may be transformed into this cyclide; and that the principal properties of the surface may be obtained geometrically by means of the transformation.

8. The relation between the radii and distance between centers giving the condition that two circles may have a simultaneously in- and circumscribed quadrilateral was obtained by various mathematicians (Fuss, Steiner, Jacobi, Cayley) in a form limited to a special case. The complete formulas are found by Dr. McDonald, incidentally giving the interpretation of certain results of the theory of elliptic functions.

9. Professor Dickson's paper appears in full in the present number of the Bulietis. W. A. Manning, Secretary of the Section.

\title{
ON QUADRATIC FORMS IN A GENERAL FIELD.
}

BY PROFESSOR L. E. DICKSON.

(Read before the San Francisco Section of the American Mathematical Society, September 28, 1907.)

1. WE investigate the equivalence, under linear transformation in a general field $F$, of two quadratic forms *

$$
q \equiv \sum_{i=1}^{n} \alpha_{i} x_{i}^{2}, \quad Q \equiv \sum_{i=1}^{n} \alpha_{i} X_{i}^{2} \quad\left(a_{i} \neq 0, \alpha_{i} \neq 0\right) .
$$

An obvious necessary condition is that $\alpha_{1}$ shall be representable by $q$, viz., that there shall exist elements $b_{i}$ in $F$ such that

$$
\alpha_{1}=\sum_{i=1}^{n} a_{i} b_{i}^{2}
$$

* Within any field $F$, not having modulus 2 , any quadratic form of nonvanishing determinant is equivalent to one of type $q$. 
We assume that this condition is satisfied. Applying a suitable permutation of the $x_{i}$, we may set $(\S 2)$

$$
W_{k} \equiv \sum_{i=1}^{k} a_{i} b_{i}^{2} \neq 0 \quad(k=1, \cdots, n) .
$$

In view of $\S 3$, the transformation

$$
x_{i}=b_{i} y_{1}+W_{i-1} y_{i}-b_{i} \sum_{j=i+1}^{n} a_{j} b_{j} y_{j} \quad(i=1, \cdots, n)
$$

is of non-vanishing determinant and replaces $q$ by

$$
q^{\prime} \equiv \alpha_{1} y_{1}^{2}+\sum_{j=2}^{n} a_{j} W_{j} W_{j-1} y_{j}^{2}
$$

By the theorem proved in $\S 4$ by a consideration of the automorphs of $q^{\prime}$, the forms $Q$ and $q^{\prime}$ (with like first coefficients) are equivalent in $F$ if, and only if,

$$
\sum_{i=2}^{n} \alpha_{i} X_{i}^{2}=\sum_{j=2}^{n} a_{j} W_{j} W_{j-1} y_{j}^{2}
$$

under a transformation in $F$ on $n-1$ variables. Hence the necessary and sufficient conditions for the equivalence of the two given n-ary quadratic forms $q$ and $Q$ are that $\alpha_{1}$ be representable by $q$ and that the $(n-1)$-ary forms $(1)$ be equivalent in $F$. The ultimate criteria are that $\alpha_{1}, \alpha_{2}, \ldots, \alpha_{n}$ be representable by forms in $n, n-1, \ldots, 1$ variables, respectively, whose coefficients are given functions of the $a_{i}$. For example, if $n=2$, the conditions are that $\alpha_{1}$ be representable by $q$ and that $a_{1} a_{2} \alpha_{1} \alpha_{2}$ be a square in $F$. If $n=3$, the conditions are that $\alpha_{1}$ be representable by $q, \alpha_{2}$ by $a_{1} \alpha_{2} W_{2} \xi^{2}+a_{3} \alpha_{1} W_{2} \eta^{2}$, and that $a_{1} a_{2} a_{3} \alpha_{1} \alpha_{2} \alpha_{3}$ be a square in $F$.

2. Theorem.* In a non-modular $\dagger$ field $F$ there exists a form

* Transactions, vol. 7 (1906), pp. 276-8. The present proof is decidedly simpler and leads to the explicit expressions (17) for the $A_{j}$, as required for the present applications.

$\dagger$ The proof applies to fields having a modulus $p$, where $p \neq 2$ and $p \geqq n$. It may be extended to apply to any finite field. To $q$ we apply the transformation

$$
x_{1}=r y_{1}+s y_{2}, \quad x_{2}=t y_{1}-t^{-1} a_{2}^{-1} r s a_{1} y_{2} \quad(t \neq 0, s \neq 0),
$$

and obtain $m y_{1}^{2}+m a_{1} s^{2} a_{2}^{-1} t^{-2} y_{2}^{2}+a_{3} x_{3}^{2}+\cdots$, where $m=a_{1} r^{2}+a_{2} t^{2} \neq 0$. By choice of $r$ and $t$, we may give $m$ any assigned value in a finite field. 
$\sum A_{i} y_{i}^{2}$ equivalent to a given form $q=\sum a_{i} x_{i}^{2}$ and having as its first coefficient $A_{1}$ any preassigned mark $\neq 0$ which is representable by $q$.

By hypothesis, there exist marks $b_{i 1}$ of $F$ such that

$$
\sum_{i=1}^{n} a_{i} b_{i 1}^{2}=A_{1} \neq 0 \text {. }
$$

Not every sum of $n-1$ of the terms $t_{i}$ of (2) vanishes. For if so, we consider the sum lacking $t_{j}$ and the sum lacking $t_{k}$ and conclude that $t_{i}=t_{k}$ and hence that the $n$ terms are all equal and that each is not zero; but this requires that $F$ shall have a modulus dividing $n-1$. By applying a permutation on the $x^{\prime}$ 's in $q$, we may set $\sum_{i=1}^{n-1} a_{i} b_{i 1}^{2} \neq 0$. Not every sum of $n-2$ terms of the latter vanishes, since $F$ does not have a modulus dividing $n-2$, etc. We may therefore set

$$
W_{k} \equiv \sum_{i=1}^{k} a_{i} b_{i 1}^{2} \neq 0 \quad(k=1, \cdots, n) .
$$

Under the transformation

$$
x_{i}=\sum_{j=1}^{n} b_{i j} y_{j} \quad(i=1, \cdots, n),
$$

$q$ becomes $\sum\left(A_{j} y_{j}^{2}+2 B_{j k} y_{j} y_{k}\right),(j, k=1, \ldots, n ; k>j)$, where

$$
A_{j}=\sum_{i=1}^{n} a_{i} b_{i j}^{2}, \quad B_{j k}=\sum_{i=1}^{n} a_{i} b_{i j} b_{i k} .
$$

To make $B_{1 k}=0$, we take

$$
b_{1 k}=-a_{1}^{-1} b_{11}^{-1} \sum_{i=2}^{n} a_{i} b_{i 1} b_{i k} \quad(k=2, \cdots, n) .
$$

We insert these values in $\Delta=\left|b_{i j}\right|$, remove the factor $a_{1}^{-1} b_{11}^{-1}$ from the first row, then multiply the $i$ th row by $a_{i} b_{i 1}$ and add to the first row, for $i=2, \ldots, n$. We get

$$
\Delta=a_{1}^{-1} b_{11}^{-1} A_{1} \Delta_{11}, \quad \Delta_{11} \equiv\left|b_{i s}\right| \quad(i, s=2, \cdots, n) .
$$

We introduce the abbreviations, in addition to (3),

$$
P_{i t}=a_{i} a_{t} b_{i 1} b_{t 1}, \quad R_{s i}=a_{i}\left(a_{i} b_{i 1}^{2}+W_{s-1}\right),
$$

so that, in particular, $R_{s s}=a_{s} W_{s}$. We find that

$$
R_{s s} R_{s i}-P_{i s}^{2}=a_{s} W_{s-1} R_{s+1 i}, \quad R_{s s} P_{i t}-P_{i s} P_{s t}=a_{s} W_{s-1} P_{i t} .
$$


Eliminating the $b_{1 k}$ from $a_{1} b_{11}^{2} B_{j k}(2 \leqq j<k)$ by $(6)$, we get

$$
B_{j k}^{(2)} \equiv \sum_{i=2}^{n} b_{i j}\left(R_{2 i} b_{i k}+\sum_{t \pm i}^{t=2, \ldots, n} P_{i t} b_{t k}\right) .
$$

Now $R_{22} \equiv a_{2} W_{2} \neq 0$. To make the coefficient of $b_{2 j}$ zero, we take

$$
b_{2 k}=-R_{22}^{-1} \sum_{t=3}^{n} P_{2 t} b_{t k} \quad(k=3, \cdots, n) .
$$

Set $b_{i 2}=0(i>2)$. Then $B_{2 k}^{(2)}$ evidently vanishes, while

$$
\Delta_{11}=b_{22} \Delta_{22}, \quad \Delta_{22} \equiv\left|b_{i s}\right| \quad(i, s=3, \cdots, n) .
$$

Generalizing (6) and (11), we shall take

$$
\begin{array}{cr}
b_{s k}=-R_{s s}^{-1} \sum_{t=s+1}^{n} P_{s t} b_{t k} & (k=s+1, \cdots, n), \\
b_{i l}=0 & (i>l>1),
\end{array}
$$

and then prove by induction from $s$ to $s+1$ that, for

$$
1<s \leqq j<k \leqq n,
$$

the product of $B_{j k}$ by a non-vanishing factor equals

$$
B_{j k}^{(s)} \equiv \sum_{i=s}^{n} b_{i j}\left(R_{s i} b_{i k}+\sum_{t \neq i}^{t=s, \ldots, n} P_{i t} b_{t k k}\right)
$$

By (10) this statement is true for $s=2$. The coefficient of $b_{s j}$ in (15) is zero by (13), so that we may set $i>s$. From the term given by $t=s$, we eliminate $b_{t k}$ by means of (13). Hence $R_{s s} B_{j k i}^{(s)}=\sum_{i=s+1}^{n} b_{i j}\left\{\left(R_{s s} R_{s i}-P_{i s}^{2}\right) b_{i k}+\sum_{t \neq i}^{t=s+1, \ldots, n}\left(R_{s s} P_{i t}-P_{i s} P_{s t}\right) b_{t k k}\right\}$.

Applying (9), we get

$$
R_{s s} B_{j k}^{(s)}=a_{s} W_{s-1} B_{j k}^{(s+1)},
$$

so that the induction is complete. Thus for given values of $j$ and $k, 1<j<k$, we may increase $s$ and make $s>j$; then (15) vanishes by (14). Hence every $B_{j k}=0(k>\hat{j})$. Finally, if 
we take each $b_{i i} \neq 0$, the determinant of (4) is not zero, in view of (14) and (7) or (12).

3. From (13), in combination with $(14)$ and $\left(9_{2}\right)$, we get

$$
b_{s k}=-a_{s}^{-.1} W_{k-1}^{-1} P_{s k} b_{l k k}
$$

Starting with the simple formulas (14) and (16), we may readily verify that, in (5), each $B_{j k}=0$, and

$$
A_{j}=a_{j} W_{j} W_{j-1}^{-1} b_{j j}^{2} \quad(j=2, \cdots, n) .
$$

TheOREM. For given elements $b_{i 1}$ satisfying (2) and (3), and any elements $b_{j j} \neq 0(j>1)$, the transformation, of non-vanishing determinant,

$$
x_{i}=b_{i 1} y_{1}+b_{i i} y_{i}-b_{i 1} \sum_{j=i+1}^{n} a_{j} b_{j 1} b_{j j} W_{j-1}^{-1} y_{j} \quad(i=1, \cdots, n)
$$

replaces $\sum a_{i} x_{i}^{2}$ by $\sum A_{j} y_{j}^{2}$, the $A_{j}$ being given by (2), (17).

If we employ the special values $b_{j j}=W_{j-1}$ and set $b_{i 1}=b_{i}$, we obtain the simpler results given in $\S 1$.

4. Within the field $F$, let the forms

$$
Q=\sum_{i=1}^{n} \alpha_{i} X_{i}^{2}, \quad E=\sum_{i=1}^{n} e_{i} y_{i}^{2} \quad\left(\alpha_{i} \neq 0, e_{i} \neq 0\right)
$$

be equivalent under the transformation

$$
S: \quad X_{i}=\sum_{j=1}^{n} \sigma_{i j} y_{j} \quad(i=1, \cdots, n) .
$$

In view of the formulas

$$
2 e_{i} y_{i}=\frac{\partial E}{\partial y_{i}}=\frac{\partial Q}{\partial y_{i}}=\sum_{j=1}^{n} 2 \alpha_{j} X_{j} \frac{\partial X_{j}}{\partial y_{i}}=2 \sum_{j=1}^{n} \alpha_{j} \sigma_{j i} X_{j},
$$

the inverse of $S$ is

$$
S^{-1}: \quad y_{i}=e_{i}^{-1} \sum_{j=1}^{n} \alpha_{j} \sigma_{j i} X_{j} \quad(i=1, \cdots, n) .
$$

Eliminating the $y_{i}$ from $Q=E$, we get

$$
\sum_{i=1}^{n} e_{i}^{-1} \sigma_{j i}^{2}=\alpha_{j}^{-1}, \quad \sum_{i=1}^{n} e_{i}^{-1} \sigma_{j i} \sigma_{k i}=0 \quad(j \neq k) .
$$


Next, let $E(y)=m E(\xi)$ under the transformation

$$
C: \quad \xi_{i}=\sum_{j=1}^{n} \gamma_{i j} y_{j} \quad(i=1, \cdots, n) .
$$

Replacing $\alpha_{j}$ by $m e_{j}$ and $\sigma_{j i}$ by $\gamma_{j i}$ in (21) and (22), we get

$$
\begin{array}{r}
C^{-1}: \quad y_{i}=m e_{i}^{-1} \sum_{j=1}^{n} e_{j} \gamma_{j i} \xi_{j} \quad(i=1, \cdots, n), \\
\sum_{i=1}^{n} e_{i}^{-1} \gamma_{j i}^{2}=m^{-1} e_{j}^{-1}, \quad \sum_{i=1}^{n} e_{i}^{-1} \gamma_{j i} \gamma_{k i}=0 \quad(j \neq k) .
\end{array}
$$

Eliminating the $y_{i}$ between (20) and (24), we obtain relations of the form

$$
X_{i}=\sum_{j=1}^{n} \beta_{i j} \xi_{j} \quad(i=1, \cdots, n),
$$

which imply $Q=m E(\xi)$. We desire that the first of these relations shall reduce to $X_{1}=\xi_{1}$. By (20), (23), the conditions are

$$
\gamma_{1 j}=\sigma_{1 j} \quad(j=1, \cdots, n) .
$$

In view of $\left(22_{1}\right)$ and $\left(25_{1}\right)$, for $j=1$, these require that $m e_{1}=\alpha_{1}$. Conversely, if $Q=E$ under $S$, and if there exists in $F$ a matrix $\left(\gamma_{i j}\right)$ with the first row identical with that of $S$ and such that $E(y)=e_{1}^{-1} \alpha_{1} E(\xi)$ under $C$, then will $Q=e_{1}^{-1} \alpha_{1} E(\xi)$ under a transformation (26) with $X_{1}=\xi_{1}$, and therefore

$$
\sum_{i=2}^{n} \alpha_{i} X_{i}^{2}=e_{1}^{-1} \alpha_{1} \sum_{i=2}^{n} e_{i} \xi_{i}^{2}
$$

under a transformation in $F$ on $n-1$ variables.*

As noted in $\S 1$, it suffices to treat the case $\nmid e_{1}=\alpha_{1}$. We have therefore to deal with automorphs of $E(y)$. In view of

* For $\sigma_{1 i}=0(i>1),\left(22_{2}\right)$ with $j=1$ gives $\sigma_{k 1}=0(k>1)$.

$\dagger$ The general case presents an essential difficulty. By $(24),\left|C^{-1}\right|=m^{n}|C|$, so that $m^{n}=\Delta^{-2}$, where $\Delta=\left|\gamma_{i j}\right|$. For $n$ odd, $m$ must therefore be a square in $F$, and hence cannot, in general, be made equal to $e_{1}^{-1} \alpha_{1}$, where $\alpha_{1}^{-1}$ is an arbitrary element of the form $\Sigma e_{i}^{-1} \sigma_{1 i}^{2}$; see $\left(22_{1}\right)$ for $j=1$. The case $n=2$ is quite simple; we may take as $\left(\gamma_{i j}\right)$ the matrix

$$
\left(\begin{array}{cc}
\sigma_{11} & \sigma_{12} \\
-e_{2}^{-1} e_{1} \sigma_{12} & \sigma_{11}
\end{array}\right), \quad e_{1}^{-1} \sigma_{11}^{2}+e_{2}^{-1} \sigma_{12}^{2}=\alpha_{1}^{-1} .
$$


$\S 5$, we can determine a matrix $\left(\gamma_{i j}\right)$ satisfying the above conditions. We therefore have the

Theorem. If $Q$ and $E$ are equivalent in $F$ under $S$ and if $e_{1}=\alpha_{1}$, then $Q$ is equivalent to $E(\xi)$ under a transformation in $F$ with $X_{1}=\xi_{1}$, so that $\sum_{i=2}^{n} \alpha_{i} X_{i}^{2}$ and $\sum_{i=2}^{n} e_{i} \xi_{i}^{2}$ are equivalent in $F$ under a transformation on $n-1$ variables.

Conversely, if the latter forms are equivalent in $F$, then evidently $Q$ and $E$, with $e_{1}=\alpha_{1}$, are equivalent in $F$.

5. Theorem. For any set of solutions $\sigma_{i}$ in $F$ of

$$
\sum_{i=1}^{n} e_{i}^{-1} \sigma_{i}^{2}=e_{1}^{-1}
$$

the quadratic form $E=\sum e_{i} y_{i}^{2}$ has an automorph in $F$ which replaces $y_{1}$ by $\sum \sigma_{j} y_{j}$.

Let $Y$ be any skew symmetric matrix, $I$ the identity (unit) matrix, $E^{-1}$ the inverse of the matrix of $E$. Let $Z=E^{-1} Y$. Then, by Cayley's theorem, $E$ has the automorph

$$
P \equiv(I+Z)^{-1}(I-Z) \quad(\Delta \equiv|I+Z| \neq 0) .
$$

It will suffice to take as $Y$ the skew matrix in which the elements outside of the first row and column are all zero, while the first row is

Hence

$$
0, c_{2}, \cdots, c_{n}
$$

$$
\begin{gathered}
I \pm Z=\left(\begin{array}{ccccc}
1 & \pm c_{2} e_{1}^{-1} & \pm c_{3} e_{1}^{-1} & \cdots & \pm c_{n} e_{1}^{-1} \\
\mp c_{2} e_{2}^{-1} & 1 & 0 & \cdots & 0 \\
\cdot \cdot & \cdot & \cdot & \cdot & \cdot \\
\mp c_{n} e_{n}^{-1} & 0 & 0 & \cdots & 1
\end{array}\right) \\
\Delta=1+\kappa, \quad \kappa \equiv e_{1}^{-1} \sum_{i=2}^{n} e_{i}^{-1} c_{i}^{2}
\end{gathered}
$$

The first row of $(I+Z)^{-1}$ is

$$
\frac{1}{\Delta}, \frac{-c_{2}}{e_{1} \Delta}, \frac{-c_{3}}{e_{1} \Delta}, \cdots, \frac{-c_{n}}{e_{1} \Delta} .
$$

Hence the first row of the product $P$ is

$$
\frac{1-\kappa}{\Delta}, \frac{-2 c_{2}}{e_{1} \Delta}, \frac{-2 c_{3}}{e_{1} \Delta}, \ldots, \frac{-2 c_{n}}{e_{1} \Delta} .
$$


These elements are to be made equal to

$$
\sigma_{1}, \sigma_{2}, \sigma_{3}, \cdots, \sigma_{n}
$$

respectively. Hence, by $\left(30_{1}\right)$, we take

$$
2=\Delta\left(1+\sigma_{1}\right), \quad \kappa=\frac{1}{2} \Delta\left(1-\sigma_{1}\right), \quad c_{i}=-\frac{1}{2} \Delta e_{1} \sigma_{i} \quad(i=2, \cdots, n) .
$$

Eliminating the $c_{i}$ from $\left(30_{2}\right)$, and applying (29), we get

$$
\kappa=\frac{1}{4} \Delta^{2} e_{1} \sum_{i=2}^{n} e_{i}^{-1} \sigma_{i}^{2}=\frac{1}{4} \Delta^{2}\left(1-\sigma_{1}^{2}\right) .
$$

Hence the conditions may be satisfied if $1+\sigma_{1} \neq 0$. But for $\sigma_{1}=-1$, we may first apply the automorph $y_{1}^{\prime}=-y_{1}$.

6. Although not employed in the present paper, the following generalization of the preceding theorem may be noted :

THeorem. Let $r$ be any positive integer $\leqq n$. If the $\sigma_{j i}$ are any solutions in $F$ of relations (22), for $j, k=1, \cdots, r$, and $a_{j}=e_{j}$, the form $E$ has an automorph in $F$ which replaces $y_{i}$ by $\sum_{j=1}^{n} \sigma_{i j} y_{j}$, for $i=1, \cdots, r$.

The proof by induction, based on the result of $\S 5$, is similar to that in the American Journal, volume 23 (1901), page 344, for the special case of a finite field with special values of the $e_{i}$.

7. Let $F^{\prime}$ be field $R$ of all rational numbers. There exist* rational values of $b_{1}, \ldots, b_{4}$ such that $\sum_{i=1}^{4} a_{i} b_{i}^{2}$ equals +1 or -1 , according as $a_{1}, \cdots, a_{4}$ are not all negative, or all negative. Hence, by $\S 1$, any $n$-ary rational quadratic form of non-vanishing determinant is reducible by a linear transformation with rational coefficients to one of the forms

$$
f_{p, a, b, c} \equiv \sum_{i=1}^{p} x_{i}^{2}-\sum_{i=p+1}^{n-3} x_{i}^{2}+a x_{n-2}^{2}+b x_{n-1}^{2}+c x_{n}^{2},
$$

in which $a, b, c$ are all negative if $p<n-3$, while $f_{p, a, b, c}$ is reducible to $f_{p, a, \beta, \gamma}$ if, and only if, $\uparrow$ the ternary form

$$
t_{a, b, c} \equiv a x^{2}+b y^{2}+c z^{2}
$$

is reducible in $R$ to $t_{\alpha, \beta, \gamma}$ (see end of $\S 1$ ).

* A simple consequence (Transactions, l. c., p. 279) of a theorem due to A. Meyer; cf., Bachmann, Zahlentheorie, $\mathrm{IV}_{1}$, p. 266.

$\dagger$ This part of the result was not given in my former paper. 